\title{
Una aproximación a los estudios autobiográficos en el Perú
}

Sandra Pinasco Espinosa

Resumen

Perú es un país que ha vivido de espaldas a su memoria. No existe una tradición de escritura autobiográfica, sino textos aislados que se han convertido en un apéndice del canon literario. Incluso en el ámbito académico, los estudios autobiográficos han sido muy poco trabajados. En este artículo quisiera mostrar, en primer lugar, que sí existen textos para ser trabajados y que con ellos se puede generar nueva bibliografía crítica. Para ello presentaré una visión panorámica de lo que podría conformar el canon autobiográfico peruano. A continuación, analizaré el estado de los estudios autobiográficos en el Perú, para finalmente discutir las diversas explicaciones que se han dado tanto a la ausencia de textos autobiográficos como al escaso interés en estudiarlos.

Palabras clave: textos autobiográficos, estudios autobiográficos en el Perú, literatura autobiográfica peruana.

Abstract

Peru is a country that has usually turned its back to its own memory. We find no proper autobiographical tradition but isolated texts that have become a sort of appendix in relation to the literary canon. Moreover, autobiographical studies are almost unheard of in the academic milieu. In this paper, I will first show that indeed there are texts to work with, and that new bibliography can be generated by presenting an overview of texts that should be considered as part of the Peruvian autobiographical canon. Then, I shall address the state of autobiographical studies in Peru, to finally discuss the various explanations given to the lack of autobiographical texts and to the limited interest in studying them.

Key words: autobiographic texts, autobiographic studies, Peruvian autobiographic literature 
G xisten múltiples problemas para el estudio de los textos autobiográficos peruanos. Uno Ude los principales es la escasa cantidad de material que ha sido considerado propiamente autobiográfico. Otro problema es la casi inexistente bibliografía sobre estos textos. De hecho, se puede contrastar esta situación con las arraigadas tradiciones autobiográficas de otros países de la región como Argentina, Chile, Colombia, Cuba o México.

En este artículo se buscará mostrar que sí existe una amplia selección de textos autobiográficos peruanos y que se puede generar nueva bibliografía. Para ello, primero se presentará un recuento de textos que pueden y deben ser considerados como parte del canon autobiográfico peruano. Luego se analizará el estado actual de los estudios autobiográficos en el Perú para, finalmente, discutir las diversas explicaciones dadas a la escasez de textos autobiográficos así como al limitado interés por estudiarlos.

\section{EI CANON AUTOBIOGRÁFICO PERUANO}

Dara poder hablar de un canon autobiográfico, resulta necesario primero aclarar algunas
ideas. En países con una larga tradición en el estudio de este género, reconstruir un canon implica por lo general salir del ámbito literario e ingresar en el terreno de los documentos históricos, de los textos de no ficción, de los testimonios, de los escritos periodísticos e incluso de manifestaciones musicales, artísticas, cinematográficas, etc. En cambio, en el caso del Perú, así como de otros países latinoamericanos, hablar de textos autobiográficos es restringirse a aquellos producidos por autores literarios, puesto que aún se conserva la idea de que la escritura autobiográfica es ancilar a la producción literaria y sirve básicamente como bibliografía secundaria para estudiar a esos mismos autores.

Una vez aclarado ese punto, los primeros textos que podrían ser considerados como parte de este canon son las crónicas coloniales. Estos textos fueron escritos mayoritariamente por extranjeros que describieron una realidad que no entendían por completo mediante el uso de categorizaciones extrańas a dicha realidad. Ahora bien, aunque en su mayoría son testimonios escritos en primera persona con una dosis de autoconciencia, estos textos no siempre han sido considerados autobiográficos (Angvik 282). Por ello básicamente han sido estudiados desde un punto de vista histórico o colonialista.

Silvia Molloy, una de los pocos académicos dedicados a investigar la tradición autobiográfica en América Latina, resume las razones por las cuales no considera que las crónicas sean propiamente autobiográficas. En primer lugar, su propósito nunca fue 
autobiográfico; además, fueron escritas como medio para obtener ciertos privilegios o favores de la autoridad a la que estaban dedicadas (13). Finalmente, la primera persona casi nunca atraviesa una crisis a lo largo de la narración (13). En resumen, las crónicas casi nunca muestran autoconfrontación, rasgo que para Molloy es central en todo texto autobiográfico (13).

No obstante se puede argumentar que muchos textos autobiográficos nunca fueron escritos con esa intención; por ejemplo, los relatos de colonos en Australia o en las colonias de las indias americanas son estudiados como textos autobiográficos y muestran muchas de las características de las crónicas, entre ellas el ser escritos que buscaban más ser testimonios de una experiencia o labor encomendada por algún superior —a la manera de una bitácora o algún otro documento que registre el paso de los días y los hechos acaecidos en los mismosque un medio para presentarse a sí mismos o sus reflexiones. De la misma manera, el que estos textos busquen lograr algún tipo de favor no significa que en la búsqueda de ese favor no estén mostrando algún tipo de análisis de sí mismos, sus vivencias o la historia que están protagonizando. Por último, la autoconfrontación no necesita ser explícita para existir; si un colono se enfrenta a una realidad inesperada para la que no tiene un nombre, el buscar ese nombre, es decir, el buscar insertar esa realidad en un marco familiar es una forma de solucionar la confrontación que existe en su sistema de creencias original al encontrarse con dicha realidad nueva.

De otro lado, algunos de los escritos coloniales han sido extensamente estudiados como la Nueva Crónica y Buen Gobierno (1615-1616); sin embargo, estos autores son estudiados solo desde una perspectiva colonialista, una aproximación teórica muy extendida en la especialidad de Literatura Hispánica de las principales universidades limeñas. Se puede mencionar como excepción el caso de Los comentarios reales de Garcilaso de La Vega, uno de los escritores coloniales peruanos más reconocidos, cuya obra ha sido estudiada desde múltiples aproximaciones teóricas y temáticas: literaria (Mazzotti 1996; Fernández 2004), histórica (Varner 1968), filosófica (Escalante 2004), religiosa (Majfud 2007), sociológica o antropológica (Valcárcel 1939; Sánchez 1979), colonial (Wachtel 1973; Zamora 1988), psicoanalítica (Hernández 1993) e incluso biográfica (Miroquesada 1994).

El siguiente periodo de la literatura peruana empieza con la Declaración de la Independencia (1821) e incluye básicamente el costumbrismo y un romanticismo tardío. De este periodo datan las 'tradiciones', género creado por Ricardo Palma, quien también es 
el primer autor peruano en escribir una pequeña autobiografía formal en 1919, el mismo año de su muerte (Cf. Porras Barrenechea 1954: 1-27).

Con el paso al siglo XX surge el Modernismo con poetas como Ruben Darío, José Martí y Leopoldo Lugones. A este movimiento perteneció también José Santos Chocano, quien escribió un texto autobiográfico al final de su vida, cuando se encontraba retirado en Chile, titulado Memorias: las mil y una aventuras (1940). De esta época también data un Diario intimo (1969) escrito entre 1914 y 1916 por José Ventura Calderón, un peruano que vivía en París y peleó en la Primera Guerra Mundial. El suyo es uno de los únicos cinco diarios conocidos publicados por peruanos ${ }^{1}$.

Para cuando el Modernismo se estaba desvaneciendo, un movimiento de vanguardia surgió en la poesía con exponentes como Cesar Vallejo, Alberto Hidalgo, Xavier Abril y Carlos Oquendo de Amat. Como parte de ese grupo de poetas, Martín Adán escribió Diario de un poeta (1966-1973), el único intento de este tipo en la poesía peruana; sobre ello reflexiona Angélica Serna Jerí en su artículo «Diario y conflicto en Diario de poeta de Martín Adán» (2004). Por otro lado, también se han analizado los aspectos autobiográficos de la poesía temprana de Vallejo (Cuadernos Hispanoamericanos, N. 456-457) e incluso algunos de los poemas de Xavier Abril o Carlos Oquendo de Amat parecieran parodiar el formato autobiográfico. Por ejemplo, en 5 metros de poemas (1927), Oquendo de Amat imita las notas biográficas de los autores presentes al final de los libros o en las contracarátulas, escribiendo en la última página de su poemario:

\section{Biografía}

tengo 19 años

y una mujer parecida a un canto

También se ha podido encontrar una biografía de este poeta escrita por Carlos Meneses (1973); así como un estudio que incluye un análisis de los aspectos autobiográficos de su obra (Ayala 1988).

1 La lista casi completa la brinda Sandra Granados (1994): «el Diario intimo (García Calderón 1969), Sobre mi propia vida. Diario (Ríos 1993), Diario de un peruano (Pareja Soldán 1978) y La tentación del fracaso (Ribeyro 1992, 1993 y 1995)». El único ausente es el diario de Alberto Jochamowitz, Lima d'antan (journal 1947-1949), publicado en 1971. Este fue un burócrata peruano que vivía en París, quien también fue responsable de la publicación del texto de García Calderón (Cf. Ribeyro, La caza sutil 119-126). 
Entre los años 1920 y 1930 se desarrolló el indigenismo, otro movimiento literario central que duró hasta 1970. Se pueden rastrear sus orígenes a escritores anteriores del siglo XIX como Narciso Aréstegui y Clorinda Matto de Turner. Más adelante se convirtió en un discurso político iniciado por Manuel Gonzáles Prada (Nuestros indios 1905) y seguido por Dora Mayer, Hildebrando Castro, José Carlos Mariátegui y Víctor Raúl Haya de la Torre.

Algunos años más tarde, otro grupo de escritores siguió las ideas del indigenismo y escribió las novelas que hoy se consideran parte del indigenismo cultural. Luis Valcarcel con Tempestad en los Andes (1927) y Enrique López Albújar con Cuentos andinos (1920) son estudiados como fundadores oficiales de este movimiento literario, seguidos por José Uriel García. Otros autores representativos de esta corriente son Ciro Alegría y José María Arguedas.

Resulta interesante señalar que este movimiento dio lugar a la creación de múltiples textos autobiográficos. López Albújar escribió De mi casona. Un poco de historia piurana a través de la biografía del autor (1924) y Memorias (1963); Alegría, Mucha suerte con harto palo: memorias (1976), editado póstumamente por su esposa; y sobre Arguedas uno podría decir que prácticamente toda su producción se encuentra inspirada autobiográficamente (Cf. Lemogodeuc 1991), aunque resalta el caso de El zorro de arriba, el zorro de abajo (1971) por presentar fragmentos del diario personal del escritor. Queda por ser estudiada la relación entre los postulados indigenistas y la opción por la escritura autobiográfica en estos autores.

El siguiente grupo de autores que se mencionará es conocido como la generación del 50. Algunos de los renombrados poetas pertenecientes a este grupo son Javier Sologuren, Sebastián Salazar Bondy, Jorge Eduardo Eielson y Blanca Varela, caracterizados como un movimiento de vanguardia que empezó a publicar en la década de 1930.

De estos, la poesía de Eielson muestra múltiples rasgos autobiográficos; por ejemplo, en su libro El cuerpo de Giulia-no (1971) «juega con fragmentos autobiográficos de un narrador en primera persona que prácticamente desaparecen confundidos con diversos momentos de las biografías fragmentadas de Giulia, Giulia-na y Giulia-no» (Angvik 293294).

De otro lado, en la narrativa de esta misma época resaltan Mario Vargas Llosa, Julio Ramón Ribeyro y Carlos Eduardo Zavaleta, todos con algún texto autobiográfico publicado. Por ejemplo, Vargas Llosa publicó El pez en el agua en 1993, una autobiografía formal que fue un éxito de ventas, aunque algunos críticos, como Birger Angvik, señalaran 
que mostraba una visión tradicional y hasta anticuada del género (Angvik 284), e incluso «una falta de lecturas, orientación e intertextos» (Angvik 299).

Por otra parte, el libro de Zavaleta, Autobiografía fugaz (2000), es de edición bastante reciente, pero no ha recibido mucha atención de los críticos. Finalmente, los diarios de Ribeyro, La tentación del fracaso: Diario personal (1950-1978), muestran un gran afecto y dominio del género, tema central de varios de sus ensayos en su libro La caza sutil (1976). Esto quizá se deba a que mantuvo un diario durante toda su vida o quizá a que durante las casi cuatro décadas que vivió en Europa se dedicó a leer todos los diarios que pudo encontrar (Cf. Fernández 1996).

La generación de 1960 está representada por poetas como Luis Hernández, Javier Heraud, Antonio Cisneros, César Calvo y Rodolfo Hinostroza. A esta generación también pertenecen los narradores Oswaldo Reynoso, Miguel Gutiérrez, Eduardo Gonzáles Viaña, Jorge Díaz Herrera y Alfredo Bryce Echenique. De todos ellos, solo Bryce Echenique ha publicado dos volúmenes de Antimemorias (vol. 1: 1993/vol. 2: 2005), título tomado del texto de André Malraux. Sobre la aproximación de Bryce a la escritura autobiográfica, se puede revisar el artículo de Mariano De Andrade (2005).

Finalmente, hace dos años se publicó la biografía de Luis Hernández (Romero 2008), luego de una investigación que aprovechó toda la documentación disponible en uno de los dos mayores archivos bibliográficos sobre un autor peruano albergados en universidades limeñas. El otro archivo está dedicado al poeta Martín Adán, pero hasta el momento su potencial biográfico no ha sido totalmente aprovechado.

\section{ESTUDIOS AUTOBIOGRÁFICOS EN EL PERÚ}

os estudios autobiográficos prácticamente no existen en el Perú. Como se ha buscado
mostrar en la primera parte de este artículo, la bibliografía primaria es escasa; así mismo, las pocas publicaciones de los últimos veinte años que analizan o investigan textos autobiográficos peruanos son, en su mayoría, superficiales. Esto se debe en parte a que solo se encuentran traducciones de algunos artículos teóricos clásicos de Gusdorf, Weintraub, De Man y Eakin, todos ellos compilados en un volumen monográfico del Suplemento Anthropos, de por sí difícil de encontrar. Por ello, como existen muy escasas contribuciones teóricas de académicos latinoamericanos y ninguna traducción de investigaciones extranjeras recientes, los pocos artículos peruanos que se aproximan a la escritura autobiográfica usan la misma bibliografía una y otra vez. 
De estos artículos, la mayoría son análisis de los hechos de la vida del autor o un contraste entre diferentes textos; también se busca establecer la naturaleza del texto autobiográfico: ¿es realmente un diario? (Fernández 2005) ¿O es solo correspondencia? ¿O quizá un «semi diario»? (Ribeyro 1976, 119). De otro lado, algunos artículos, como el de Del Castillo (1994), buscan explicar la falta de interés por lo autobiográfico en el Perú.

Finalmente, se han publicado múltiples libros sobre los autores canónicos enumerados en la primera parte del artículo: Garcilaso, Guamán Poma, Oquendo de Amat, Vallejo, Hernández, Ribeyro, Bryce or Vargas Llosa, pero solo algunos trabajan específicamente el tema autobiográfico. Uno de ellos es la investigación que Angvik dedica a la labor de los críticos literarios peruanos de los siglos XIX y XX, en la cual dedica un capítulo a la autobiografía. El otro es la investigación de Silvia Molloy, aunque solo se aproxima a la producción peruana de forma tangencial. Específicamente le dedica tres páginas a Mi casona de López Albújar (227-229) y menciona el primer capítulo de las memorias de Chocano, «El hombre que no fue niño» (1940), con una cita de las primeras líneas (Molloy 146). El único trabajo peruano contemporáneo es el libro El Perú en la memoria. Sujeto y nación en la escritura autobiográfica (2007) de Cecilia Esparza. Producto de su tesis doctoral, Esparza analiza los textos autobiográficos de Santos Chocano, López Albújar y Arguedas, así como los de Ribeyro, Vargas Llosa y Bryce. No obstante, este trabajo necesita ser aún explotado en nuevas investigaciones.

Desde una perspectiva diferente, solo se han sustentado ocho tesis universitarias que trabajen temas autobiográficos desde 1940, dos de ellas recuentos laudatorios de la persona como tema (Alva Kont 1940; Weksler Farace 1989). De las otras seis, una es una tesis de maestría en antropología que se centra en el arte popular, específicamente en uno de los muchos artesanos peruanos (Balarín 1996). La más reciente es de la Facultad de Arte y trabaja las formas en que la iconografía presenta a un personaje histórico famoso (Quezada 2007). Dos más se concentran en personajes históricos, uno del periodo colonial y otro del conflicto armado con Colombia en 1933; ambas son contribuciones bastante recientes (Miranda 2002; Merino 2004). Finalmente, solo dos pertenecen a la especialidad de Lingüística y Literatura, una es una aproximación narratológica al discurso autobiográfico en El zorro de arriba y el zorro de abajo de Jose Maria Arguedas (Torres 2002) y la otra es una investigación de la autobiografía ficcional presente en otra de las novelas de Arguedas, Los ríos profundos. Como ya ha sido mencionado, este es uno de los pocos autores peruanos investigados en diversas ocasiones desde un punto de vista autobiográfico. 
Asimismo, desde una aproximación psicológica, las autobiografías son usualmente analizadas para crear patografías de sus autores. Este tipo de investigación está basada, por lo general, en las memorias del autor, así como en cartas o entrevistas a los parientes y amigos cercanos, siempre que resulte posible. El problema con esta aproximación es que el material es trabajado como un recuento indiscutible de los hechos con el cual se busca reconstruir la vida del autor, en lugar de una reconstrucción que busque resignificar esos mismos hechos.

De otro lado, la falta de publicaciones que se concentren en una temática autobiográfica puede también estar relacionada con que los estudiantes no reciben la oportunidad de trabajar con estos textos. Sin embargo, existe un creciente interés por los temas autobiográficos, especialmente desde una aproximación historiográfica o sociológica. Por ejemplo, a los estudiantes de Historia y de Ciencias Sociales se les enseñan algunas herramientas para trabajar con narraciones de vidas, aunque por lo general traten la información obtenida solo como un dato o documento, en lugar de como una narración factible de ser analizada desde otros puntos de vista. Asimismo, como existen diversas investigaciones antropológicas dedicadas a las tradiciones orales quechua o aimara, así como a diversas culturas de la región amazónica, prácticamente todos los estudiantes de las especialidades de Ciencias Sociales deben realizar trabajo de campo para acopiar material de primera mano de las comunidades indígenas. Asimismo, autores como Cristina Sáenz de Tejada, con su investigación La (re)construcción de la identidad femenina en la narrativa autobiográfica latinoamericana, 1975-1985 (1988), o Carlos Pacheco con su artículo Textos en la frontera: autobiografía, ficción y escrituras de mujeres (1999) muestran que el enfoque de género puede ser otra forma de aproximarse a la escritura autobiográfica latinoamericana.

Por último, la única conferencia peruana dedicada a la escritura autobiográfica, titulada «Escribir de sí mismo. Historia y autodocumentos en los Andes» (26 y 27 de febrero de 2009), fue organizada en el Instituto Raúl Porras Barrenechea conformado mayoritariamente por historiadores. En esta conferencia, Sebastián Chávez Wurm, de la Universidad de Hamburgo, presentó una ponencia sobre los testimonios de terroristas recogidos por la Comisión de la Verdad y la Reconciliación para analizar sus características autobiográficas. De hecho, parece existir un creciente interés por los testimonios en Sudamérica, tal como muestran artículos como el de Adlin de Jesús Prieto Rodríguez, «Del testimonio a la autobiografía. Aproximación a Aqui no ha pasado nada (1972) de Ángela Zago» (2005), aunque ese mismo escrito muestra cómo el carácter autobiográfico de los testimonios aún se encuentra en discusión por quienes se aproximan a la escritura 
autobiográfica desde una perspectiva ortodoxa largamente superada por la comunidad académica internacional. Esta misma perspectiva parece ser la que guía otro proyecto, titulado «Del testimonio a la autobiografía. Ángela Zago y su proyecto de escritura», alrededor de la producción de esta misma autora, quien escribió diversos testimonios acerca de sus experiencias en las FALN, una guerrilla organizada por el partido comunista para acabar con el gobierno de Betancourt.

Ahora, en el programa de mano de esta única conferencia, se especifican sus fuentes primarias como 'autodocumentos'. Este concepto muestra que los investigadores participantes se han aproximando al material autobiográfico básicamente como documentos históricos que hablan del pasado en lugar de considerarlos como material válido para un análisis del discurso, o un trabajo interdisciplinario alrededor de los aspectos narrativos, o para el análisis de los efectos que la escritura del texto tuvo en su autor o la función cultural que el texto cumple.

Esto se ve confirmado con la diferencia que se establece entre el análisis histórico de los 'autodocumentos' y la aproximación literaria a estos mismos textos. De acuerdo con el programa del congreso, «En la ciencia literaria se discute un gran número de definiciones de género, mientras que la pregunta más importante para la historiografía es: ¿qué nos dicen los autodocumentos del pasado?». De hecho, consideran que estos «documentos» explican cómo su autor entendió su tiempo y cultura, así como la manera en que interactuó como miembro de un contexto sociocultural determinado. Por ello, al contrastar estos «documentos» se puede enriquecer la visión y comprensión del pasado.

De esta oposición entre los investigadores literarios como dedicados exclusivamente a establecer la «naturaleza» del género autobiográfico frente a los historiadores, sociólogos y antropólogos que investigan lo que estos textos trasmiten, pueden surgir algunas explicaciones sobre el por qué los investigadores peruanos muestran tan poco interés en los estudios autobiográficos.

\section{ALGUNAS RESPUESTAS A IAS PREGUNTAS PLANTEADAS}

Como se ha postulado a lo largo del artículo, cuando uno busca estudiar la producción autobiográfica peruana, uno necesita contestar las siguientes preguntas: ¿por qué existe tan poca bibliografía primaria? ¿Por qué existe tan poco interés y conocimiento sobre el tema autobiográfico? Ya desde 1953, Julio Ramón Ribeyro buscó aclarar estas interrogantes basándose en el carácter del peruano, en cierta forma tímido, introvertido y dispuesto a 
defender su vida privada de la opinión pública.

Solo cabe formular hipótesis: falta de tradición, poca capacidad introspectiva, conciencia inmadura de la propia persona, menosprecio por un tipo de obra cuya repercusión es generalmente póstuma y, en último término, ¿por qué no? concepción machista de la literatura, que hace considerar la redacción de un diario como cosa de seńoritas. (Ribeyro 1976, 119)

Sin embargo, caben otras respuestas. La primera explicación, centrada en la idiosincrasia del peruano, y por ello semejante a la del ensayo de Ribeyro, es que las sociedades latinoamericanas son mayoritariamente católicas. Considerando que la escritura autobiográfica fue un resultado principalmente del protestantismo, debido a que «este movimiento religioso, con su teoría del libre examen, favoreció la técnica de la introspección y el nacimiento de la noción de la persona» (Ribeyro 1976: 13) como un ser independiente; la presencia del catolicismo podría ser una razón por la cual el género autobiográfico no ha desarrollado raíces demasiado profundas en la cultura latinoamericana. Sin embargo, esta afirmación no explica por qué la situación en Perú es tan diferente en relación con países vecinos en relación con países vecinos como Argentina o Chile donde sí es factible encontrar una tradición autobiográfica desarrollada en ámbitos urbanos. En todo caso, el argumento del catolicismo podría afirmarse de realidades más rurales que son profundamente religiosas y que usan la oralidad como el mejor instrumento para preservar la memoria, pero no aplica para contextos urbanos.

Más adelante, en una entrevista dada algunos años antes de su muerte en 1992, Ribeyro señaló que había cambiado su forma de pensar en relación a sus ensayos de 1953 y que quizá el problema era que Latinoamérica simplemente no contaba con una tradición de escritura autobiográfica. "Mientras no existan diarios íntimos, mientras sus autores no comiencen a publicar sus diarios íntimos, pues otros no lo harán. Entonces, es una cuestión de ir sentando una tradición» (Coaguila 1996: 52-53).

Otra explicación ha sido que la distancia entre las esferas pública y privada nunca ha estado bien establecida. A lo largo de la historia del Perú, y de muchos otros países latinoamericanos, las dictaduras militares y la inseguridad policial han interferido siempre con la vida ciudadana. En cierta medida, parecía que no hubiese un derecho a la historia privada o un "desencuentro entre subjetividad e historia» (del Castillo 38), entre el sentido público y el privado (38), entre las tramas individual y colectiva (39), esto es, entre lo 
individual y lo colectivo (53-54). Para Daniel del Castillo, el origen de este desacuerdo es la culpa y la mala conciencia siempre presentes en la tradición política y cultural peruana, así como la sensación de ilegitimidad que gobierna la conciencia peruana (47-48). Sin embargo, se podría responder que esta explicación está basada en una simple dicotomía, un «desencuentro esencial y esencialista» (Angvik 329) y aislaría al Perú de su contexto sudamericano, porque nuevamente no explica los resultados tan disímiles entre países que comparten una misma tradición de gobiernos militares y dictaduras.

También en su libro sobre la crítica literaria peruana, así como en diferentes entrevistas, Angvik llega a una conclusión interesante: la tendencia de los críticos peruanos a buscar significados autobiográficos en los textos literarios «...y dónde está la referencia autobiográfica. Y si no hay, no sale. En César Vallejo insisten en la autobiografía» (entrevistado por Giancarlo Stagnaro, 2005). Este rasgo de los críticos peruanos puede ser interpretado como una forma de cubrir la escasez de escritos autobiográficos en la producción literaria peruana, o como la razón por que los autores obvian las referencias autobiográficas para así poder evitar esas interpretaciones, o simplemente como una visión reaccionaria de la literatura.

Una tercera explicación para la falta de textos autobiográficos en el Perú es que quizá los peruanos simplemente no quieren recordar. La historia peruana nunca ha sido una de éxitos o riqueza, ¿por qué alguien quisiera recordarla? Recién en los últimos ocho a diez años, con una economía creciente y un panorama político más estable, los autores más jóvenes parecieran estar buscando nuevas formas de expresión, esto incluye escribir sobre sí mismos de forma más o menos explícita. Así Internet cuenta con herramientas como el Facebook o los blogs que permiten a cualquiera la oportunidad de contar su historia, aunque este es un fenómeno reciente en el área editorial y se deberá esperar un tiempo para evaluar sus resultados.

En esa misma dirección, Molloy considera que las autobiografías latinoamericanas han sido, por lo general, leídas como ficción o historia, dependiendo del discurso hegemónico de su tiempo. De ahí que «el lector, al negar al texto autobiográfico la recepción que merece, solo refleja, de modo general, una incertidumbre que ya está en el texto, unas veces oculta y otras evidente» (12). Eso puede ser porque, hasta el siglo XIX, los textos autobiográficos buscaban dar testimonio de su realidad y, para lograrlo, los autores usaban la narración de sus vidas como prueba de ser testigos de su tiempo. Quizá también por eso una buena parte de los textos autobiográficos latinoamericanos evita lidiar con recuerdos de infancia, 


\section{SANDRA PINASCO ESPINOSA}

así como con cualquier reflexión acerca de la memoria y las formas en que esta trabaja o, peor aún, falla. «La memoria se suele aceptar como eficaz mecanismo de reproducción cuyo funcionamiento rara vez se cuestiona, cuyas infidelidades apenas se prevén» (Molloy 18). Siguiendo estas reflexiones, uno podría generalizar que la tradición autobiográfica peruana no se ha desarrollado, porque todavía existe una confusión entre un recuento histórico de hechos y la experiencia personal de esos mismos hechos. 


\section{Bibliografía}

Alva Kont, Marcela

1940 Perfiles de la vida y obra de Manuel Ascencio Segura. Tesis para optar al grado de Bachiller en la Facultad de Letras y Ciencias Humanas en la sección de Lengua y Literatura. Lima: Fondo Editorial de la Pontificia Universidad Católica del Perú.

ANGvik, Birger

1999 La ausencia de la forma da forma a la crítica que forma el canon literario peruano. Lima: Fondo Editorial de la Pontificia Universidad Católica del Perú.

Ayala, José Luis

1988 Carlos Oquendo de Amat. Cien metros de biografia, critica y poesía de un poeta vanguardista itinerante. De la subversión semántica a la utopía social. 1. a ed. Lima: Editorial Horizonte.

Balarín Benavides, Claudia

1996 El arte de usar las manos: Santiago Rojas y el arte popular. Tesis para optar al grado de Magíster en Antropología. Lima: Fondo Editorial de la Pontificia Universidad Católica del Perú.

Coaguila, Jorge

1996 Ribeyro. La palabra inmortal. Entrevistas con Jorge Coaguila. Lima: Jaime Campodónico editor.

De Andrade, Mariano

2005 «Permiso para inventar: Bryce y la autobiografía». Quehacer, N. 157, noviembrediciembre, pp. 120-125. Lima.

Del Castillo, Daniel

1994 «Lo autobiográfico en el Perú contemporáneo». Márgenes: encuentro y debate. N. 12, noviembre, pp. 37-54. Lima. 
Escalante, Marie Elise

2004 Un estudio sobre la nominación en las crónicas de Garcilaso de la Vega y Guamán Poma. Lima: Fondo editorial de la Universidad Nacional Mayor de San Marcos.

Esparza, Cecilia

2007 El Perú en la memoria. Sujeto y nación en la escritura autobiográfica. Lima: Red para el Desarrollo de las Ciencias Sociales en el Perú.

FERnÁNDEZ, Christian

1996 «Autobiografía e identidad en La tentación del fracaso». En MazzotTi, José Antonio. Asedios a la heterogeneidad cultural: libro de homenaje a Antonio Cornejo Polar. Filadelfia: Asociación Internacional de Peruanistas.

2004 Inca Garcilaso: Imaginación, memoria e identidad. Lima: Fondo editorial de la Universidad Nacional Mayor de San Marcos.

2005 «El zorro de abajo y el zorro de arriba (1971): ficción o autobiografía». El Hablador, revista electrónica, N. 5, septiembre. Disponible en <www.elhablador.com/zorro. htm>.

Gonzáles Prada, Manuel

1977 Discurso en el Politeama; Grau; Nuestros indios. Lima: Universo.

Granados, Sandra

2004 «El estatuto ribeyriano del diario». El Hablador, revista electrónica, N. 6, diciembre. Disponible en <www.elhablador.com/estatuto.htm>.

HERnÁNDEZ, Max

1993 Memorias del bien perdido. Conflicto, identidad y nostalgia en el Inca Garcilaso de la Vega. Lima: Instituto de Estudios Peruanos/Biblioteca Peruana de Psicoanálisis.

Cuadernos Hispanoamericanos

1998 Homenaje a César Vallejo. Cuadernos Hispanoamericanos, Número monográfico, N. 456-457, junio-julio. Madrid. 
Lemogodeuc, Jean Marie

1991 «Significado y riesgos del realismo autobiográfico en la obra de José María Arguedas». En Forgues, Roland; Hildebrando Pérez y Carlos Garayar (eds.). José María Arguedas: Vida y obra. Lima: Amaru Editores.

López Albújar, Enrique

1970 Cuentos andinos. Lima: Juan Mejía Baca.

MajFud, Jorge

2007 «Mestizaje cosmológico y progreso de la historia en el Inca Gracilaso de la Vega». Araucaria. Revista Iberoamericana de Filosofía, Politica y Humanidades, año 9, N. 18, segundo semestre.

Mazzotti, José Antonio

1996 Coros mestizos del Inca Garcilaso. Resonancias andinas. Lima: Fondo de Cultura Económica.

Meneses, Carlos

1973 Tránsito de Carlos Oquendo de Amat (1905-1936). (Biografía del autor). Las Palmas de Gran Canaria: Inventarios Provisionales.

Merino Amand, Marco Antonio

2004 Fernando Lores Tenazoa: el heroismo de un joven soldado y provinciano. Tesis para optar al grado de Licenciado en la Facultad de Letras y Ciencias Humanas con mención en Historia. Lima: Fondo Editorial de la Pontificia Universidad Católica del Perú.

Miranda Larco, Giuliana

2002 El pensamiento de Fray Luis Jerónimo de Oré en el Symbolo Católico Indiano y en el Ritvale sev Manuales Pervanvm (1554-1630). Tesis para optar al grado de Licenciado en la Facultad de Letras y Ciencias Humanas con mención en Historia. Lima: Fondo Editorial de la Pontificia Universidad Católica del Perú. 
Molloy, Silvia

1991 At Face Value. Autobiographical Writing in Spanish America. Nueva York: Cambridge University Press.

1996 Acto de presencia. La escritura autobiográfica en Hispanoamérica. Traducción de José Esteban Calderón. México: El colegio de México/ Fondo de Cultura Económica.

Pacheco, Carlos

1999 «Textos en la frontera: autobiografía, ficción y escrituras de mujeres». En KoнUT, Kart (ed.). Literatura venezolana hoy. Historia nacional y presente urbano. Frankfurt/ Main-Madrid: Americana Eystettensia, pp. 127-137.

Porras Barrenechea, Raúl

1954 «De la autobiografía a la biografía de Palma». Letras Peruanas, N. 4, 1954, pp. 1-27.

Portugal Flaherty, Jose Alberto

1984 Autobiografía y autobiografía ficcional en Los ríos profundos de Jose María Arguedas. Tesis para optar al grado de Bachiller en la Facultad de Letras y Ciencias Humanas en la especialidad de Lingüística y Literatura. Lima: Fondo Editorial de la Pontificia Universidad Católica del Perú.

Prieto Rodríguez, Adlin de Jesús

2005 «Del testimonio a la autobiografía. Aproximación a Aqui no ha pasado nada (1972) de Ángela Zago». El Hablador, revista electrónica, N. 5, setiembre, disponible en $<$ www.elhablador.com/zago.htm>.

Quesada Orellana, Mario Nicolás

2007 Esbozos iconológicos acerca de "El último cartucho». Tesis para optar al grado de Licenciado en la Facultad de Arte con mención en Pintura. Lima: Fondo Editorial de la Pontificia Universidad Católica del Perú.

Ribeyro, Julio Ramón

1976 La caza sutil. Lima: Milla Batres. 
Romero Tassara, Rafael

2008 La armonía de H. Vida y poesía de Luis Hernández Camarero. Lima: Jaime Campodónico.

SÁENZ de Tejada, Cristina

1998 La (re)construcción de la identidad femenina en la narrativa autobiográfica latinoamericana, 1975-1985. Nueva York: Peter Lang.

SÁNCHEZ, Luis Alberto

1979 Garcilaso Inca de la Vega, primer criollo. [1939]. 5. a y definitiva ed. Lima: Fondo del Libro del Banco de los Andes.

Serna Jerí, Angélica

2004 «Diario y conflicto en Diario de poeta de Martín Adán». El Hablador, revista electrónica, N. 3, marzo. Disponible en <www.elhablador.com/adan.htm>.

StAGNARO, Giancarlo

2005 «Birger Angvik exorciza a Mario Vargas Llosa». Entrevista. Identidades: reflexión, arte y cultura, suplemento del diario oficial El Peruano, edición N. 80, 7 de marzo del 2005. Disponible en <www.editoraperu.com.pel identidades/80/entrevista.asp $>$.

\section{Suplemento Anthropos}

1981 Monographic number. It compiles translations of key theoretical articles on autobiography, N. 9 .

Torres Vitolas, Miguel Ángel

2002 Los recursos veridictorios del discurso autobiográfico en los diarios de El zorro de arriba y el zorro de abajo. Tesis para optar al grado de Licenciado en la Facultad de Letras y Ciencias Humanas con mención en Lingüística. Lima: Fondo Editorial de la Pontificia Universidad Católica del Perú.

VALCÁRCEL, Luis

1939 Garcilaso el Inca, visto desde el ángulo indio. Lima: Imprenta del Museo Nacional.

1970 Tempestad en los Andes. Lima: Populibros Peruanos. 
VARNer, John Grier

1969 El Inca: The Life and Times of Garcilaso de la Vega. Austin: University of Texas Press.

WACHTEL, Nathan

1973 «Pensamiento salvaje y aculturación: el espacio y el tiempo en Felipe Guamán Poma de Ayala y el Inca Garcilaso de la Vega». Sociedad e ideología: ensayos de historia y antropología andinas. Lima: Instituto de Estudios Peruanos, pp. 161-228.

WeKsler Farace, Marina Eva

1989 Fernando de Szyszlo: aproximaciones a su vida y a su obra. Tesis para optar al grado de Bachiller en la Facultad de Arte. Lima: Fondo Editorial de la Pontificia Universidad Católica del Perú.

ZAGO, Angela

1972 Aqui no ha pasado nada. Caracas: Síntesis Dos mil.

ZAMORA, Margarita

1988 Language, Authority and Indigenous History in the "Comentarios Reales de los Incas". Cambridge: Cambridge University Press.

\section{Bibliografía DE TEXTOS AUTOBiográFICOS PERUANOS}

ADÁn, Martín

1980 Diario de poeta. 1966-1973. Obra Poética. Fundación del Banco Continental para el fomento de la Educación y la Cultura. Lima: Edubanco, 1980.

Alegría, Ciro

1976 Mucha suerte con harto palo: memorias. Buenos Aires: Losada.

Arguedas, José María

1971 El zorro de arriba y el zorro de abajo. Buenos Aires: Editorial Losada.

1990 El zorro de arriba y el zorro de abajo. Ed. Eve-Marie Fell. Madrid: Colección Archivos. 
Bryce Echenique, Alfredo

1999 Permiso para vivir. Antimemorias. Lima: Peisa.

2005 Permiso para sentir. Antimemorias 2. Lima: Peisa.

Chocano, José Santos

1940 Memorias: las mil y una aventuras. Santiago de Chile: Nascimento.

De la Vega, Garcilaso

2007 Comentarios Reales de los Incas. Lima: Fondo Editorial Universidad Inca Garcilaso de la Vega.

2008 Segunda parte de los Comentarios Reales de los Incas. 2 vols. Lima: Fondo Editorial Universidad Inca Garcilaso de la Vega.

EiELSON, Jorge Eduardo

1971 El cuerpo de Giulia-no. México, D.F.: Joaquín Mórtiz.

García Calderón, José

1970 Diario intimo. (12 de setiembre, 1914 - 3 de mayo, 1916). Lima: Imprenta de la Universidad Nacional Mayor de San Marcos.

Guamán Poma de Ayala, Felipe

1993 Nueva Corónica y buen gobierno. 3 vols. México, D.F.: Fondo de Cultura Económica.

IgARTÚA, Francisco

1995 Siempre un extraño. Vol. 1. Lima: Santillana.

1998 Huellas de un destierro. Vol. 2. Lima: Aguilar.

JochaмOwitz, Alberto

1973 Lima d'antan (journal 1947-1949). Paris: Imprimerie S.I.P.E.

López Albújar, Enrique

1996 De mi casona. Un poco de historia piurana a través de la biografía del autor. Lima: Juan Mejía Baca.

1963 Memorias. Lima: P. L. Villanueva. 
OQuendo de Amat, Carlos

20075 metros de poemas. (Edición facsimilar del original de 1927). Lima: Editorial Universitaria de la Universidad Ricardo Palma.

Palma, Ricardo

194(?) Autobiografía de Ricardo Palma. Leyendas y tradiciones indigenas. Luis Eduardo Valcárcel. Selección realizada por Camila Estremadoyro Robles. Lima: s. e.

2005 Epistolario general. tr., pról., notas e índices Miguel Ángel Rodríguez Rea. 2 vols. Lima: Editorial Universitaria de la Universidad Ricardo Palma.

Ribeyro, Julio Ramón

2003 La tentación del fracaso: diario personal (1950-1978). Barcelona: Seix Barral.

Ríos, Juan

1993 Sobre mi propia vida. Diario 1940-1991. Derechos reservados Juan Ríos, SUC. Se terminó de imprimir en febrero de 1993 en los talleres gráficos de Graph Service Cosmos. (Edición personal del autor).

VArgas Llosa, Mario

2005 El pez en el agua. Madrid: Alfaguara.

Zavaleta, Carlos Eduardo

2000 Autobiografía fugaz. Lima: Fondo editorial de la Universidad Nacional Mayor de San Marcos.

Esta recopilación de textos autobiográficos peruanos no se considera concluida. Cualquier colaboración a la misma será bienvenida a la siguiente dirección de correo: spinasco@uarm. edu.pe 